\title{
Multiple Factorial Analysis of Symbolic Data
}

\author{
Barnabé Tang Ahanda, Jean Gérard Aghoukeng Jiofack, \\ Romain Germain Nzangué, Gilbert Hapi Mbiakop \\ Department de Mathematics, Faculty of Sciences, University of Yaounde 1, Yaounde, Cameroon \\ Email: tangahanda@yahoo.fr, aghoukeng-maths@yahoo.fr
}

Received September 10, 2012; revised November 13, 2012; accepted November 20, 2012

\begin{abstract}
This document presents an extension of the multiple factorial analysis to symbolic data and especially to space data. The analysis makes use of the characteristic coding method to obtain active individuals and the reconstitutive coding method for additional individuals in order to conserve the variability of assertion objects. Traditional analysis methods of the main components are applied to coded objects. Certain interpretation aids are presented after the coding process. This method was applied to poverty data.
\end{abstract}

Keywords: Symbolic Object; Assertion Object; Multiple Factorial Analysis; Principle Components Analysis; Poverty

\section{Introduction}

Although the traditional analysis of data is based on a complete theory, it poses restrictive conditions for the resolution of problems: it requires that variables be single-valued (the value of a variable for an object is a unique and specific).

Recent evolutions in the information system have made it possible to find increasing and more complex data which need a more in-depth formalization than the one indicated in the usual rectangular table. The later does not take into account the possible variability of description as well as the uncertainty concerning certain variables or certain objects. A new formalism called symbolic objects has been developed to represent complex data like concepts, skills (see $[1,2]$ ). These objects concern complex data and provide new skills at the exit in the form of symbolic objects.

The techniques of factorial analysis (see $[3,4]$ ) which are data reduction and representation methods, are well known and well used. Certain stakeholders have extended these methods to symbolic objects by using the coding and decoding techniques (read $[5,6])$. The issue of multiple factorial analysis (MFA) is of particular interest to us $($ see $[7,8])$. A priori, this concerns the case where there is a group structure on variables. Its problem is enhanced by the characterization of groups and the search for a typology of groups of variables. To our knowledge, this method is not yet applied to symbolic objects.

In this article, we extend the MFA to a symbolic data base whose variables are structured into groups. It is a generalization of the MFA basic principle on the one hand, and the principle components analysis (PCA) tech- niques on the other hand (see [9]). We conclude this document with an application of the method presented to poverty data.

\section{Symbolic Data Base}

\subsection{General Context}

A symbolic data base (SDB) is considered as represented in the Table 1 and made up of triplet $B=\{Y, C, A\}$ with $C=\left\{C_{1}, C_{2}, \cdots, C_{I}\right\}$ representing all the $I$ objects, $Y=\left\{y_{1}, y_{2}, \cdots, y_{J}\right\}$ and $A=\left\{a^{1}, a^{2}, \cdots, a^{I}\right\}$ representing respectively the set of the $J$ variables and the assertion objects used to describe $I$ objects. We suppose that the SDB is associated with a measured space $(\Omega, \mathcal{F}, \mu)$, an $\sigma$-algebra $\mathcal{C}$ on $C, O=\left\{O_{1}, O_{2}, \cdots, O_{J}\right\}$ where

$O_{j}$ is the space of observation of the variable

$y_{j}, j=1,2, \cdots, J$. This space includes an tribe $\mathcal{O}_{j}$. Let $\left(X_{c_{i}, j}\right)_{j=1,2, \cdots, J}$ be a set of measurable functions defined on $\Omega$, deriving their value from the measurable spaces $\left(O_{j}, \mathcal{O}_{j}\right)$ and used to describe all the $c_{i}$ objects. For $i$ from 1 to $I, a^{i} a^{i}=\bigwedge_{j=1}^{J}\left[y_{j}=X_{a^{i}, j}\right]$ is a Boolean assertion so that from $j$ from 1 to $J$, there is a measurable part $V_{a^{i}, j} \in \mathcal{O}_{j}$ so that we can have

$X_{a^{i}, j}=X_{c_{i}, j}$ and $V_{a^{i}, j}=X_{a^{i}, j}(\Omega)$. In practice, it is written

$$
a^{i}=\bigwedge_{j=1}^{J}\left[y_{j}=V_{a^{i}, j}\right] .
$$


Table 1. Symbolic data base.

\begin{tabular}{cccccc}
\hline & \multicolumn{1}{c}{$y_{1}$} & & & $y_{J}$ \\
\hline$c_{1}$ & {$\left[y_{1}=X_{a^{1}, 1}\right]$} & $\ldots$ & {$\left[y_{j}=X_{a^{1}, j}\right]$} & $\ldots$ & {$\left[y_{J}=X_{a^{1}, J}\right]$} \\
$c_{i}$ & {$\left[y_{1}=X_{a^{i}, 1}\right]$} & $\ldots$ & {$\left[y_{j}=X_{a^{i}, j}\right]$} & $\ldots$ & {$\left[y_{J}=X_{a^{i}, J}\right]$} \\
$C_{I}$ & {$\left[y_{1}=X_{a^{l}, 1}\right]$} & $\ldots$ & {$\left[y_{j}=X_{a^{I}, j}\right]$} & $\ldots$ & {$\left[y_{J}=X_{a^{l}, J}\right]$} \\
\hline
\end{tabular}

The observation space is therefore the product space $\Theta_{j}=\prod_{j=1}^{J} O_{j}$. This space includes an product tribe $\Xi_{j}=\prod_{j=1}^{J} \mathcal{O}_{j}$. To proceed, it is supposed that the descriptors are quantitative, i.e that $O_{j}$ is a part of $\mathbb{R}$. Thus, it is generalized that $X_{a^{i}, j}(\Omega)=V_{a^{i}, j}=\left[x_{i j}, \overline{x_{i j}}\right]$ where $x_{i j}, \overline{x_{i j}}$ represent respectively the minimum and maximum values per $a^{i}$ object for the $y_{j}$ variable. Consequently, we shall treat the Boolean assertions whose SDB is indicated in the following Table 2.

\subsection{Group Structure}

We shall assume that the $J$ variables are divided into $K$ groups with $J_{k}$ as the number of $k$ group variables; $p_{i}$ the weight associated with the assertion object $a^{i}$ and $m_{\ell}$ the weight of the $y_{\ell}$ variable. We shall designate $I, K, J_{k}$ as the set and its cardinal. Let us consider $B_{k}=\left\{Y_{k}, C, A_{k}\right\}$ as the symbolic data base derived from $B$ but reduced from the $k$ group variables denoted $Y_{k}$. For $i$ from 1 to $I$, the $a^{i}$ object for the $k$ group is represented by the assertion $a_{k}^{i}=\bigwedge_{j \in J_{k}}\left[y_{j}=V_{a_{k}^{i}, j}\right]$. Formula (1) may be re-written as follows, while taking into consideration the existence of the following groups:

$$
\begin{aligned}
a^{i}= & \bigwedge \\
j \in J_{1} & {\left[y_{j}=V_{a^{i}, j}\right] \bigwedge \ldots \bigwedge_{j \in J_{k}}\left[y_{j+\sum_{r<k} j_{r}}=V_{a_{k}^{i}, j+\sum_{r<k} j_{r}}\right] } \\
& \bigwedge \ldots \bigwedge_{j \in J_{K}}\left[y_{j+\sum_{r<K} j_{r}}=V_{a_{K}^{i}, j+\sum_{r<K} j_{r}}\right]
\end{aligned}
$$

\subsection{Objective}

Like in the traditional case, the MFA based on symbolic data corresponds to the following three majors objectives:

1) Drawing up a typology of concepts, especially similar concepts from the point of view of all the variables after balancing the contribution of each group.

2) Comparing the typologies of individuals defined by groups of variables.

3) Exposing a typology of groups of variables and interpreting the proximities between them.

\begin{tabular}{|c|c|c|c|c|c|}
\hline & $y_{1}$ & & $y_{j}$ & & $y_{J}$ \\
\hline$c_{1}$ & {$\left[x_{11}, \overline{x_{11}}\right]$} & $\ldots$ & {$\left[x_{1 j}, \overline{x_{1 J}}\right.$} & $\ldots$ & {$\left[\underline{x_{1 J}}, \overline{x_{1 J}}\right.$} \\
\hline$c_{i}$ & {$\left[\underline{x_{i 1}}, \overline{x_{i 1}}\right]$} & $\ldots$ & {$\left[x_{i j}, \overline{x_{i j}}\right]$} & $\ldots$ & {$\left[\underline{x_{i j}}, \overline{x_{i J}}\right.$} \\
\hline$c_{I}$ & {$\left[x_{I 1}, \overline{x_{I 1}}\right]$} & $\ldots$ & {$\left[x_{I j}, \overline{x_{I J}}\right]$} & $\ldots$ & {$\left[\underline{x_{I J}}, \overline{x_{I J}}\right.$} \\
\hline
\end{tabular}

Table 2. Symbolic data base of boolean assertions.

However, taking into consideration the variability of objects adds an interesting element to this problem because coherence warrants us to provide exit elements in keeping with the variability.

\section{Analysis and Interpretation}

We shall present algorithm for a symbolic digital MFA. This approach essentially repeats the one used by the traditional MFA as well as that of the PCA on symbolic objects. The following stages shall be used:

1) Data coding;

2) PCA of groups;

3) Weighting of the data table;

4) PCA symbolic digital of all data;

5) Traditional MFA representation and interpretation;

6) Superimposed representation of symbolic objects and average cloud;

7) Representation of assertions of variables and symbolic factors of groups

\subsection{Data Coding}

Either $\Delta$ all the assertions in the SDB, the $m$ coding order is any $C$ application to $\Delta$ with values in $\left(\mathbb{R}^{j}\right)^{m}$. In this work, we shall therefore use the centre and summit methods which is referred to as characteristic coding and reconstitutive coding respectively.

An assertion object is graphically represented by a hypercube. The characteristic coding represents each object by a point which is the centre of gravity of the assertion object. The reconstitutive coding represents an object by $2^{J}$ points which constitute the summit of the hypercube. Characteristic coding gives us the active elements of the analysis while reconstitutive coding gives the additional elements of the analysis that enable the reconstitution of the exit symbolic objects.

\subsection{Weighting of Variables}

Let $X$ be the data table obtained after data coding. A $J$ columns and $I \times\left[1+2^{J}\right]$ lines table is obtained where $I$ individuals are active and the others in addition. For each group, a principle components analysis (PCA) on the $X_{k}, k=1 \cdots K$ tables where $X_{k}$ is the $X$ table reduced to the $k$ group value. A series of 
$\lambda_{i}^{k}, i=1, \cdots, k, k=1, \cdots, K$. eigenvalues is obtained for each analysis. It is assumed that the real values in each group are classified in descending order. Each $y_{\ell}$ variable is weighted by $\frac{m_{\ell}}{\lambda_{1}^{k}}$ if the $y_{\ell}$ variable is derived from the $k$ group. The weighted table is evaluated $X^{(p)}$.

\subsection{Data Processing}

Weighting conserves the internal structures of each group but changes the structure of the overall cloud (see [10]). In this case, it is referred to as the average cloud. As in the traditional case, we have represent equivalent objects by respective lines of the same $K$ bases $\tilde{B}_{k}^{(p)}$ rank, with $B_{k}^{(p)}$ being the weighted $B_{k}$ base.

$$
\tilde{B}_{k}^{(p)}=\begin{array}{|l|l|l|}
\hline 0 & B_{k}^{(p)} & 0
\end{array} \Rightarrow \tilde{X}_{k}^{(p)}=\begin{array}{|l|l|l|}
\hline 0 & X_{k}^{(p)} & 0 \\
\hline
\end{array}
$$

The $N_{j}^{k} K$ clouds may be simultaneously represented in the $\mathbb{R}^{J}$ space of variables by breaking down $\mathbb{R}^{J}$ into direct sum spaces isomorphic to $\mathbb{R}^{j_{k}}$ spaces.

The average cloud is obtained by a homothetic transformation of the $B^{(p)} 1 / K$ relation weighted data base. It represents average individuals and average concepts in $\mathbb{R}^{k}$. The average cloud is obtained by a homothetic transformation of the $B^{(p)} 1 / K$ relation weighted data base. It represents average individuals and average concepts in $\mathbb{R}^{k}$. Let us note $B^{*}$ the SDB of the average cloud where $B_{k}^{*}$ constitutes its restriction in the $k$ group.

Proposition 1. The symbolic digital PCA of the $B^{*}$ base leads to the same factors as the $B$ base characteristic coding MFA.

Proof. It is immediate. The $B^{*}$ base is constructed to this effect.

\subsection{Canonic Variable}

The re-writing of table $X^{*}$ in the principle components base $\left(F_{1}, \cdots, F_{J}\right)$ leads to a representation of objects by a new $\operatorname{SDB} B=\left\{F, O, A_{F}\right\}$ whose assertion is written $a_{F}^{i}=\bigwedge_{j=1}^{J}\left[F_{j}=W_{a_{F}^{i}, j}\right]$. Assertions of the variables associated with concepts are written $F^{j}=\bigwedge_{i=1}^{I}\left[F_{j}=W_{a_{F}^{i}, j}\right]$. These factors are called canonic variables.

\subsection{Superimposed Representation of Individuals and Concepts}

\subsubsection{Equivalent Assertions}

A $B^{(p)}$ based $a^{i}$ assertion is represented in each $B_{k}^{(p)}$ sub-base by $a_{k}^{i}$ assertions which are called equivalent assertions associated with $a^{i}$. These are the different representatives in the $B^{(p)}$ sub-bases of the same assertion.

\subsubsection{Superimposed Representation of Individuals}

Here, individuals are described by the characteristic coding. An individual representing an object is represented by the expectation of unpredictable random. variable associated with the skill it represents. This representation no doubt has some shortcomings, but it gives us the first tendencies which are sometimes enough. In concrete terms, each group is projected with additional elements on the PCA of table $X^{*}$.

Proposition 2. Each individual (average individual) of table $X^{*}$ is the centre of gravity of individuals observed from the point of view of all groups.

Proof. This is the property of traditional MFA. $\square$

This proposition explains the notion of average cloud. The study of the attributes of variability of description indicated below will comfort us in this notion.

\subsubsection{Superimposed Representation of Concepts}

Symbolic digital PCA results will be used to obtain a superimposed representation of objects while taking into consideration variability. It based to the following proposition:

Proposition 3. Either $a^{i}=\bigwedge_{j=1}^{J}\left[y_{j}=V_{a^{i}, j}\right]$ an assertion of BDS $B$. We note $b^{i}=\bigwedge_{k=1}^{K}\left[u_{k}=V_{b^{i}, k}\right]$ the assertion representing the $c_{i}$ objects in the space of the PCA principle components of this SDB. Let $G=\left(X_{1}^{G}, \cdots, X_{J}^{G}\right)$ the centre of gravity of the characteristic coding and $u_{j k}$ the $k$ th component of the jth factorial axis. Thus we have:

$$
\begin{aligned}
\min \left(W_{b^{i}, k}\right) & =\sum_{j, u_{k j}<0}\left[\max \left(V_{a^{i}, j}\right)-X_{j}^{G}\right] u_{k j} \\
& +\sum_{j, u_{k j}<0}\left[\min \left(V_{a^{i}, j}\right)-X_{j}^{G}\right] u_{k j} \\
\max \left(W_{b^{i}, k}\right) & =\sum_{j, u_{k j}<0}\left[\min \left(V_{a^{i}, j}\right)-X_{j}^{G}\right] u_{k j} \\
& +\sum_{j, u_{k j}<0}\left[\max \left(V_{a^{i}, j}\right)-X_{j}^{G}\right] u_{k j}
\end{aligned}
$$

Proof. We note $\left(x_{i j}^{G}\right)_{j=1, \cdots, J}$ the coordinate of $G^{i}$ centre of gravity of $a^{i}$ assertion, and $\left(y_{k j}^{G}\right)_{j=1, \cdots, J}$ the coordinate of the image of $G^{i}$ in the new basis. Let $u_{j k}$ be the jth component of the kth factorial axis (denoted $\left.u_{k}\right)$, we note $x_{r}^{i}=\left(x_{i j}^{r}\right)_{j=1, \cdots, J}$ the coodinate of an elementary individual of $a^{i}$ assertion for a point $R^{i}$, and $y_{r}^{i}=\left(y_{k j}^{r}\right)_{j=1, \cdots, J}$ his coordinate in the new basis. We have:

$$
y_{k j}^{r}=\overrightarrow{\boldsymbol{G R}} \cdot u_{k}=\sum_{j=1}^{J}\left(x_{i j}^{r}-x_{i j}^{G}\right) u_{j k}
$$


then

$$
\begin{aligned}
& \max \left(W_{b^{i}, k}\right)=\max \left\{y_{k i}^{r} / x_{i j}^{r} \in V_{a^{i}, j}\right\} \\
= & \max \left\{\left[\sum_{j=1}^{J}\left(x_{i j}^{r}-x_{i j}^{G}\right) u_{j k}\right], x_{i j}^{r} \in V_{a^{i}, j}\right\}
\end{aligned}
$$

as $y_{k j}^{r}$ depend of $x_{i j}^{r}$, we have

$$
\begin{aligned}
\max \left(W_{b^{i}, k}\right)= & \sum_{j, u_{k j}<0}\left[\left(\min \left\{x_{i j}^{r}, x_{i j}^{r} \in V_{a^{i}, j}\right\}\right)-X_{j}^{G}\right] u_{k j} \\
& +\sum_{j, u_{k j}>0}\left[\left(\max \left\{x_{i j}^{r}, x_{i j}^{r} \in V_{a^{i}, j}\right\}\right)-X_{j}^{G}\right] u_{k j}
\end{aligned}
$$

Therefore

$$
\begin{aligned}
\max \left(W_{b^{i}, k}\right) & =\sum_{j, u_{k j}<0}\left[\min \left(V_{a^{i}, j}\right)-X_{j}^{G}\right] u_{k j} \\
& +\sum_{j, u_{k j}>0}\left[\max \left(V_{a^{i}, j}\right)-X_{j}^{G}\right] u_{k j} .
\end{aligned}
$$

With the similar methode, we have

$$
\begin{aligned}
\min \left(W_{b^{i}, k}\right) & =\sum_{j, u_{k j}<0}\left[\max \left(V_{a^{i}, j}\right)-X_{j}^{G}\right] u_{k j} \\
& +\sum_{j, u_{k j}>0}\left[\min \left(V_{a^{i}, j}\right)-X_{j}^{G}\right] u_{k j} .
\end{aligned}
$$

This lead that the $B_{k}$ representing group $k$ will be projected successively with additional elements on the $B^{*}$ base symbolic digital PCA. The superimposed representation on the same factorial plan enables us to generally observe the position of an object, and the point of view of each group by using variability. We then have the following result :

Proposition 4. Either $a^{i}$ an assertion object represented in $B^{*}$ by $\left(a^{i}\right)^{*}$. Let us note $a_{k}^{i}$ the $a^{i}$ assertion observed from the $k$ group point of view. In the $B^{*}$ base, the $a^{i}$ assertion is written

$$
\begin{aligned}
a^{i} & \bigwedge_{j \in J_{1}}\left[y_{j}=\frac{1}{J \sqrt{\lambda_{1}}} V_{a_{1}^{i}, j}\right] \\
& \wedge \ldots \bigwedge_{j \in J_{k}}\left[y_{j+\sum_{r<k} j_{r}}=\frac{1}{J \sqrt{\lambda_{k}}} V_{a_{k}^{i}, j+\sum_{r<k} j_{r}}\right] \\
& \wedge \ldots \bigwedge_{j \in J_{K}}\left[y_{j+\sum_{r<K} j_{r}}=\frac{1}{J \sqrt{\lambda_{K}}} V_{a_{K}^{i}, j+\sum_{r<K} j_{r}}\right]
\end{aligned}
$$

Either $a^{i}=\bigwedge_{j=1}^{J}\left[y_{j}=V_{a^{i}, j}^{*}\right]$ we note $a_{k}^{i}=\bigwedge_{k=1}^{K}\left[F_{k}=W_{a_{k}^{i}, k}\right]$ the assertion representing the $c_{i}$ object from the $\mathrm{k}$ group point of view in the space of the
PCA principle components of this SDB. Either $G=\left(X_{1}^{G}, \cdots, X_{J}^{G}\right)$ the centre of gravity of $X^{*}$, and $F_{k j}$ the $k$ th component of the jth factorial axis. Thus we have:

$$
\begin{aligned}
\min \left(W_{a_{F}^{i}, k}\right)= & \sum_{j \in J_{k}, F_{k j}<0}\left[\max \left(J V_{a^{i}, j}^{*}\right)-X_{j}^{G}\right] F_{k j} \\
& +\sum_{j \in J_{k}, F_{k j}>0}\left[\min \left(J V_{a^{i}, j}^{*}\right)-X_{j}^{G}\right] F_{k j} \\
\max \left(W_{a_{F}^{i}, k}\right)= & \sum_{j \in J_{k}, F_{F_{j}<0}<}\left[\min \left(J V_{a^{i}, j}^{*}\right)-X_{j}^{G}\right] F_{k j} \\
& +\sum_{j \in J_{k}, F_{k j}>0}\left[\max \left(J V_{a^{i}, j}^{*}\right)-X_{j}^{G}\right] F_{k j}
\end{aligned}
$$

Proof. This is the consequence of the notations of the previous proposition.

The representation of superimposed objects in the canonic base (that of factors) therefore respects the principle of centres of gravity. The hypercube representing an object is thus the centre of gravity of the hypercubes representing the same individual from the point of view of different groups. The MFA symbolic digital algorithm helps to preserve this essential property.

\subsection{Representation of Variables and Assertions of Variables}

The symbolic digital MFA enables us to define many representatives that contribute to the illustration of factors in the general analysis as well as in the different groups. The determination of the main factors of groups and the general analysis lead to many representations of variables and assertions of variables. The superimposed representation of these factors (as assertions of variables) on canonic variables then helps to find and interpret common factors and specific factors.

\subsubsection{Representation of Variables and Assertions of Variables}

The conduct of the MFA symbolic digital pre-supposes $K+1$ factorial analysis (the $K$ groups and the average cloud). Two representations can be made for each of these analyses: a representation of variables (dual analysis of centres of gravity) and an analysis of assertions of variables taking into account variability. These representations clearly bring out the main variability factors of groups and the factors of general analysis.

\subsubsection{Superimposed Representation of Factors (as Assertions of Variables) on Canonic Variables}

This representation is the result of a MFA traditional representation which consists in highlighting the main variability factors of groups in the form of additional variables over canonic variables. But in symbolic data analysis, these variability factors are also expressed as 
assertions of variables and the representation of these assertions of variables over the canonic variables calls for an original observation. The position and collection of assertions of variables give us precious indications on their importance and the link between the different groups.

\subsubsection{Link between Groups: Representation Quality of Groups}

This is a traditional indicator in MFA. It is a representation of groups on canonic factors. The coordinates of an axis group is the combined inertia of the group's variables on the corresponding MFA axis of table $X^{*}$. For the two groups $K_{x}$. and $K_{y}$, we have

$\mathcal{L}\left(K_{x}, K_{y}\right)=\left\langle W_{x}^{(p)} D, W_{y}^{(p)} D\right\rangle$ where $D$ is the diagonal $I \times I$ matrix of the weight of individuals and $W_{j}^{(p)}$ is the matrix of scalar products between the individuals of group $j$ for the $X_{j}^{p}$ weighted matrix.

A symbolic representation may be proposed for it. Each assertion of variables can be coded into many variables whose projection over canonic variables results in a minimum value and a maximum value. The variability associated to a group will therefore be displayed by two values: the sum of the minimum inertia of the group's variables and the sum of its maximum inertia. The representation of groups is done in the $\mathbb{R}^{I^{2}}$ space.

\subsection{The Notion of Common Factor and Specific Factor}

MFA helps to portray the characteristics of groups compared to the canonic variables (also referred to as factors). A common (or specific) factor to the MFA symbolic digital will be the common factor associated to the traditional MFA of centres of gravity.

\section{Poverty Data}

\subsection{Presentation}

The data is drawn from the first Cameroonian survey in households (ECAM I) conducted in 1996 by the National Institute of Statistics. The sample is made up of 1800 households with a total of 10230 individuals. The analysis is done on 13 continuous variables out of which 10 are active and 3 illustrative (see Table 3 ).

The average poverty line is used to establish the difference between poor and non poor individuals. We shall consider 10 classes of individuals with 5 poor classes organized in descending order from very poor to less poor individuals and 5 classes of non poor individuals organized in ascending order from less rich to very rich individuals. These classes constitute the concepts that we shall analyze. The groups of variables are regions. For presentation purposes, they shall be presented on a line and not in columns. An overview of the SDB for the Adamawa Region for three variables shows the following Table 4.

\subsection{Analysis and Interpretation}

After the algorithm application, the following results are obtained:

\subsubsection{Inertia of the First Six Eigenvalues Please see Table 5.}

\subsubsection{Relationship between General Variables and Groups}

The $F_{1}$ variable is the variable which extracts the most important inertia from all groups. The other variables convey specific information to certain regions (see Table 6).

Table 3. Variables of ECAM1.

\begin{tabular}{|c|c|c|c|}
\hline $\mathrm{N}$ & Title & Description & Quality \\
\hline 1 & DEPEAD & Expenditure per adult equivalent & Additional \\
\hline 2 & DEPTET & Expenditure per head & Additional \\
\hline 3 & DEPTOT & Total expenditure & Additional \\
\hline 4 & DEPALI & Food expenditure & Active \\
\hline 5 & DEPHAD & Clothing expenditure & Active \\
\hline 6 & DEPMAI & Household expenditure & Active \\
\hline 7 & DEPSAN & Health expenditure & Active \\
\hline 8 & DEPTRA & Transportation expenditure & Active \\
\hline 9 & DEPEDU & Education expenditure & Active \\
\hline 10 & DEPSOP & Personal health care expenditure & Active \\
\hline 12 & DEPLOY & Rent expenditure & Active \\
\hline 13 & DEPLOG & Lodging expenditure & Active \\
\hline
\end{tabular}


Table 4. An overview of SDB of ECAM1.

\begin{tabular}{cccc}
\hline & & AD & DEPMAI \\
\hline Concept & DEPALI & DEPHAB & {$[3950,36,000]$} \\
Poor of class 1 & {$[96,464,173,375]$} & {$[2000,36,000]$} & {$[28,800,34,800]$} \\
Poor of class 2 & {$[122,375,573,832]$} & {$[15,000,31,200]$} & {$[10,800,28,800]$} \\
Poor of class 3 & {$[109,500,573,032]$} & {$[5700,46,800]$} & {$[7200,15,300]$} \\
Poor of class 4 & {$[157,210,302,428]$} & {$[5700,38,200]$} & {$[12,000,53,700]$} \\
Poor of class 5 & {$[126,967,358,482]$} & {$[0,54,500]$} & {$[2450,55,100]$} \\
Non poor of class 1 & {$[102,982,536,028]$} & {$[0,115,900]$} & {$[11,950,57,600]$} \\
Non poor of class 2 & {$[220,303,568,617]$} & {$[0,82,000]$} & {$[23,000,65,100]$} \\
Non poor of class 3 & {$[95,421,1,707,678]$} & {$[3500,297,300]$} & {$[600,71,300]$} \\
Non poor of class 4 & {$[146,782,967,510]$} & {$[8000,485,200]$} & {$[22,800,199,800]$} \\
\hline
\end{tabular}

Table 5. Histogram of eigenvalues.

\begin{tabular}{cccc}
\hline $\mathrm{N}$ & Eigenvalue & Inertia $\%$ & Combined \% \\
\hline 1 & 6.80938 & 68.09 & 68.09 \\
2 & 1.08859 & 10.89 & 78.98 \\
3 & 0.60479 & 6.05 & 85.03 \\
4 & 0.56497 & 5.65 & 90.68 \\
5 & 0.31109 & 3.11 & 93.79 \\
6 & 0.29054 & 2.91 & 96.69 \\
\hline
\end{tabular}

Table 6. Relationship between general variables and groups.

\begin{tabular}{|c|c|c|c|c|c|c|}
\hline & $F_{1}$ & $F_{2}$ & $F_{3}$ & $F_{4}$ & $F_{5}$ & $F_{6}$ \\
\hline $\mathrm{AD}$ & 52.70 & 20.95 & 5.81 & 3.94 & 1.15 & 1.31 \\
\hline $\mathrm{CE}$ & 77.57 & 9.29 & 0.83 & 0.93 & 0.35 & 0.26 \\
\hline ES & 58.61 & 4.60 & 9.72 & 8.58 & 0.96 & 4.04 \\
\hline EN & 62.35 & 10.50 & 3.13 & 5.98 & 2.81 & 1.40 \\
\hline LT & 82.74 & 2.18 & 1.31 & 1.93 & 1.09 & 0.45 \\
\hline NO & 51.03 & 6.12 & 7.38 & 8.56 & 10.40 & 4.10 \\
\hline NW & 67.16 & 8.38 & 2.91 & 5.74 & 1.36 & 3.81 \\
\hline $\mathrm{OU}$ & 46.08 & 10.14 & 6.78 & 9.28 & 6.78 & 5.22 \\
\hline SU & 57.94 & 13.26 & 5.23 & 2.32 & 2.02 & 3.20 \\
\hline SW & 57.84 & 12.42 & 11.17 & 3.22 & 1.13 & 2.62 \\
\hline
\end{tabular}

\subsubsection{Inter-Inertial/Total Inertia Relationship}

Table 7 shows that on the first axis, there is a close proximity between equivalent classes. Broadly speaking, these equivalent classes have similar behaviours in their expenditure habits. The same phenomenon is observed in the descending values of $r$, on the 4th, 2nd, 6th and 3rd axes.

\subsubsection{Degree of Resemblance between Groups and General Variables: Common and Specific Factors}

The first MFA component is a factor common to all the 
Table 7. Inter-inertial/total inertia relationship.

\begin{tabular}{ccccccc}
\hline Axis & $F_{1}$ & $F_{2}$ & $F_{3}$ & $F_{4}$ & $F_{6}$ \\
\hline Relationship & 0.89 & 0.70 & 0.64 & 0.70 & 0.30 \\
\hline
\end{tabular}

Table 8. Degree of resemblance between groups and general variables.

\begin{tabular}{|c|c|c|c|c|c|c|}
\hline & $F_{1}$ & $F_{2}$ & $F_{3}$ & $F_{4}$ & $F_{5}$ & $F_{6}$ \\
\hline $\mathrm{AD}$ & 0.87 & 0.58 & 0.65 & 0.13 & 0.05 & 0.09 \\
\hline $\mathrm{CE}$ & 0.90 & 0.12 & 0.03 & 0.45 & 0.01 & 0.02 \\
\hline ES & 0.90 & 0.15 & 0.31 & 0.66 & 0.15 & 0.80 \\
\hline EN & 0.98 & 0.75 & 0.45 & 0.50 & 0.18 & 0.38 \\
\hline LT & 0.98 & 0.28 & 0.11 & 0.33 & 0.02 & 0.08 \\
\hline $\mathrm{NO}$ & 0.92 & 0.42 & 0.44 & 0.32 & 0.59 & 0.32 \\
\hline NW & 0.97 & 0.57 & 0.12 & 0.78 & 0.28 & 0.35 \\
\hline $\mathrm{OU}$ & 0.88 & 0.42 & 0.43 & 0.60 & 0.28 & 0.62 \\
\hline SU & 0.93 & 0.47 & 0.60 & 0.55 & 0.28 & 0.70 \\
\hline SW & 0.96 & 0.68 & 0.72 & 0.30 & 0.15 & 0.16 \\
\hline
\end{tabular}

10 regions. The second factor is common to the FarNorth and South-West regions. The 3rd factor is specific to the South-West and Adamawa regions (see Table 8).

\section{REFERENCES}

[1] E. Diday, "Introduction to the Symbolic Data Analysis," Cahiers du CEREMADE, No. 8823, 1988.

[2] E. Diday, "From the Objects of the Data Analysis to Those of the Analysis of Knowledge, Symbolic and Numeric Induction from Data," Cépadues, 1991.

[3] L. Lebart, A. Morineau and Mr. Piron, "Multidimensional Exploratory Statistics," 3rd Edition, DUNOD, Paris, 2000.

[4] M. Volle, "Data Analysis," Economica, Paris, 1981.

[5] J. F. Martin, "Fuzzy Coding and Its Applications in Sta- tistics," Thesis, University of Pau, Pau, 1980.

[6] F. J. Gallego, "Fuzzy Coding in Correspondence Analysis," Cahiers de l'Analyse des Données, Vol. 13, No. 2, 1980, pp. 413-430.

[7] J. Pagès and B. Escofier, "Simple and Multiple Factorial Analysis,” DUNOD, Paris, 1990.

[8] B. Escofier and J. Pajes, "Multiple Factorial Analysis, Objectives, Methodology and Interpretation," 2nd Edition, Wiley, Paris, 2005.

[9] P. Cazes, A. Chouakria, E. Diday and Y. Schektman, "Extension of Principal Component Analysis to Interval Data," Revue de Statistique Appliquée, Vol. 45, No. 3, 1997, pp. 5-24.

[10] B. Ahanda Tang, "Extension of Factorial Analysis to Symbolic Data," Thesis, University of Paris, Paris, 1998. 